\title{
Tumor mutation burden predicts response and survival to immune checkpoint inhibitors: a meta-analysis
}

\author{
Linghong Wan ${ }^{1,2,3}$, Zhi Wang ${ }^{1,2,3}$, Jinmin Xue ${ }^{1,2,3}$, Huaju Yang ${ }^{1,2,3}$, Yuxi Zhu ${ }^{1,2,3}$ \\ ${ }^{1}$ Department of Oncology, The First Affiliated Hospital of Chongqing Medical University, Chongqing, China; ${ }^{2}$ Department of Oncology, Jinshan \\ Hospital of The First Affiliated Hospital of Chongqing Medical University, Chongqing, China; ${ }^{3}$ Chongqing Clinical Cancer Research Center, \\ Chongqing, China \\ Contributions: (I) Conception and design: All authors; (II) Administrative support: Y Zhu; (III) Provision of study materials or patients: L Wan, Z \\ Wang; (IV) Collection and assembly of data: L Wan, Z Wang, H Yang; (V) Data analysis and interpretation: L Wan, J Xue; (VI) Manuscript writing: \\ All authors; (VII) Final approval of manuscript: All authors. \\ Correspondence to: Yuxi Zhu. Department of Oncology, The First Affiliated Hospital of Chongqing Medical University, Chongqing, China. \\ Email: zhuyuxi17@163.com; zhuyuxi@hospital.cqmu.edu.cn.
}

Background: Cancer is one of the world's top three causes of death now. Immune checkpoint inhibitors (ICIs) show encouraging ability to treat some malignancies due to its long-term efficacy and low side effects. However, the predictive biomarker of the immunotherapy efficacy has been inconclusive. Thus, exploring new biomarkers is important.

Methods: A meta-analysis was conducted to evaluate whether tumor mutation burden (TMB) could be a predictive biomarker of the efficacy of ICIs. Using the PubMed and Cochrane Library databases, we searched for articles about TMB and the prognosis of patients with multiple malignancies conducted from 1984 to May 22, 2020. We identified the relationship between TMB and the clinical efficacy of ICIs by using Stata 12.1 software.

Results: Eighteen articles with a total of 4,535 patients were included in this meta-analysis. Results showed that high-TMB patients had better progression-free survival (PFS) than low-TMB patients with cancer treated with ICIs $(\mathrm{HR}=0.45$; 95\% CI: 0.36-0.56, $\mathrm{P}=0.002)$. Moreover, high-TMB patients had longer overall survival (OS) than low-TMB patients. However, the heterogeneity was extremely high, so the result regarding $\mathrm{OS}$ was meaningless ( $\mathrm{HR}=0.56$; 95\% CI: 0.44-0.70, $\mathrm{P}=0.000$, I-squares: $72.6 \%$ ).

Conclusions: Our study indicates that high TMB is associated with better PFS. Thus, TMB can be considered as a predictive marker of PFS of patients treated with ICIs in the future.

Keywords: Tumor mutation burden (TMB); immune checkpoint inhibitors (ICIs); predictive biomarker; metaanalysis

Submitted Feb 21, 2020. Accepted for publication Aug 05, 2020.

doi: $10.21037 /$ tcr-20-1131

View this article at: http://dx.doi.org/10.21037/tcr-20-1131

\section{Introduction}

Cancer is one of the world's top three causes of death (1). In 2018, approximately 18.1 million new cancer cases and 9.1 million deaths occurred worldwide (2). Although great progress has been made in chemotherapy, radiotherapy, targeted therapy, and surgery, the clinical outcome of some kind of cancers is still poor. In recent years, checkpoint inhibitors have attracted considerable interest due to their efficacy in treating non-small cell lung cancer (NSCLC), melanoma, and lymphoma. Clinical trials have shown that immune checkpoint inhibitors (ICIs) can be used to improve the treatment of these kinds of cancers along with chemotherapy and targeted therapy (3).

ICIs, including programmed death protein 1 (PD-1) inhibitors, programmed cell death ligand 1 (PD-L1) 
inhibitors, and cytotoxic T-lymphocyte antigen 4 (CTLA-4) inhibitors, exhibit consistent efficacy, less side effects. And ICIs have no cross resistance with chemoradiotherapy. Patients with NSCLC can benefit from ICIs when they lack active mutations such as ROS-1 and EGFR (4). However, the response rate of ICIs is approximately $15-40 \%$ in most cancers (5). Thus, the characteristics of the population who might benefit from ICIs must be evaluated. The immunochemistry staining of PD-L1 in cancers might be a biomarker, and the PD-L1 expression might indicate the efficacy of ICIs. However, this method has some limitations. Some clinical trials indicate that the high expression of PD-L1 is not correlated with good prognosis $(6,7)$. Therefore, other predictive biomarkers of the efficacy of ICIs should be developed.

Tumor mutation burden (TMB) is the number of somatic mutations after removing germline mutations from the cancer genome. It contains the total number of mutations of substitution, insertion, and deletion per megabase pair in the coding exons. It is also expressed as the total number of non-synonymous mutations. In recent years, many studies have revealed that high TMB is associated with better prognosis in cancer patients receiving ICIs $(8,9)$, indicating that TMB might be a new predictive biomarker for cancers treated with ICIs. On the other hand, some studies showed that the TMB level is not related to patient prognosis (10). Therefore, we conducted a meta-analysis to explore the relationship among TMB, ICIs, and cancer prognosis.

We present the following article in accordance with the PRISMA reporting checklist (available at http://dx.doi. org/10.21037/tcr-20-1131).

\section{Methods}

\section{Literature search}

We carried out a systematic search of the medical literature in PubMed and Cochrane library until May 22, 2020, with subject headings and free words search method. The following keywords were used: TMB, tumor mutation burden, immunotherapy, PD-1, PD-L1. We also manually checked the reference cited in the identified articles.

\section{Inclusion and exclusion criteria}

The inclusion criteria: (I) the subjects of study were malignant tumor cases confirmed by pathologic diagnosis; (II) the relationship between the level of TMB and the clinical outcome of patients was described; (III) at least one or more main outcome indicators [overall survival (OS) and progression-free survival (PFS)] were available; (IV) an English language restriction was imposed. The exclusion criteria: (I) a review, case report, animal or cell line study; (II) the case number of the study was less than 30; (III) without hazard ratios (HR) and $95 \%$ confidence interval (CI) of TMB and related clinical outcome indicators. If two or more trials were included in one study with different outcomes, the data would be extracted as independent studies.

\section{Data extraction}

Data extraction was conducted by 2 reviewers independently. The following data were extracted from the eligible studies: (I) the basic information of study: first author name, year of publication, etc.; (II) tumor type, sample size; (III) TMB level standard; (IV) HR and 95\% CI of TMB and related clinical outcome indicators (if univariate and multivariate analysis were both used in the article, HR and 95\% CI were obtained by multivariate analysis).

\section{Statistical analysis}

All statistical analyses were performed using Stata 12.1. We computed HR of high TMB group and low expression group as effect index. The point estimation and $95 \% \mathrm{CI}$ of each effect size were given. The heterogeneity between studies was estimated by Chi-square test and considered to indicate no statistical heterogeneity among studies when $\mathrm{I}^{2}<50 \%$ and $\mathrm{P}>0.10$, so we combined analysis with fixed effect model. If $\mathrm{P} \leq 0.10$ or $\mathrm{I}^{2} \geq 50 \%$, there were statistical heterogeneities among the studies and the random effect model was used for combined analysis. If there was a significant heterogeneity among the results of each study, subgroup analysis or sensitivity analysis should be used to explore the source of heterogeneity. We would conduct the subgroup analysis and regression analysis to explore the source of high heterogeneity. Publication bias was assessed through egger's test. A P value $<0.05$ was judged as statistically significant.

\section{Results}

\section{Study selection}

A total of 1,096 studies were identified by our initial search in PubMed and Cochrane Library. Among them, 1,050 studies were excluded after perusing the title and 
1,096 citations searched in PubMed [830] and Cochrane [266] according to search strategy

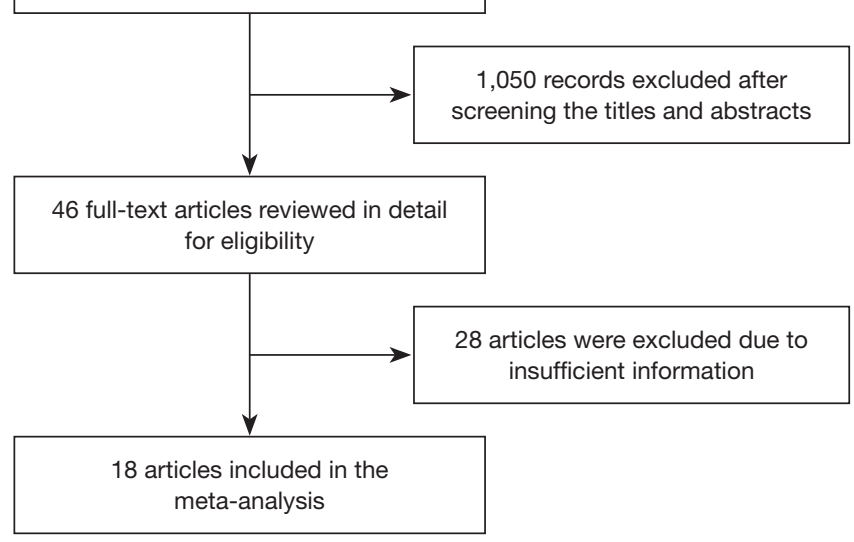

Figure 1 Literature search and selection.

abstract, and we obtained 46 primary screening articles. The remaining 46 articles were reviewed for more detailed evaluation. Thirteen of them were excluded because we were unable to obtain specific HR and 95\% CI, and 1 was excluded because we could not obtain the number of cases. Another three studies were excluded as we could not determine the Newcastle-Ottawa Scale criterion. Moreover, three, one, and five studies were excluded as they were single-arm studies, focused on predictive TMB, and did not have enough cases (less than 30), respectively. Another three studies were excluded as they focused on blood TMB. Finally, 18 studies were included in our meta-analysis, which contained 40 groups and a total of 4,535 cases that met our inclusion criteria (10-27). The selection process and results of the studies are shown in Figure 1.

\section{Characteristics of the selected studies}

Eighteen studies were published in 2015-2020, and their details and characteristics are provided in Table 1.

\section{Meta-analysis}

\section{Meta-analysis for PFS}

On the basis of the pooled result from 2,258 cases who were treated with ICIs in 16 groups of thirteen studies, which reported the relationship between TMB level and PFS, we found that tumor patients with high TMB had better PFS compared with those with low TMB (HR $=0.45 ; 95 \%$ CI: $0.36-0.56, \mathrm{P}=0.002$, I-squared $=57.7 \%)$. The difference was statistically significant (Figure 2).

\section{Meta-analysis for OS}

Among the included literatures, 12 studies reported the relationship between TMB level and OS. The difference in OS between cancer patients with high TMB and those with low TMB was statistically significant according to the analysis result (HR $=0.56$; 95\% CI: 0.44-0.70, $\mathrm{P}=0.000$, I-squares: $72.6 \%$ ) (Figure 3). However, we found that the $\mathrm{I}^{2}$ value was very high, indicating a great heterogeneity that cannot be ignored. Thus, the result was meaningless. To analyze the source of heterogeneity, we made a subgroup analysis according to the types of cancer. The results showed that NSCLC patients with high TMB had longer OS than those with low TMB (HR $=0.50 ; 95 \%$ CI: $0.38-0.64, \mathrm{P}=0.295$, I-squarely: $18.3 \%$ ) (Figure 4). For other cancers, some of the data were insufficient or had great heterogeneity. Thus, subgroup analysis could not be performed. We conducted a subgroup analysis of the types of ICIs and found that patients with high TMB who received atezolizumab had longer OS than those with low TMB (HR $=0.66$; 95\% CI: 0.47-0.93, P=0.398, I-square $=0$ ) (Figure 5). To explore whether the types of cancer and ICIs are sources of heterogeneity, we conducted a regression analysis and found that they were not sources of high heterogeneity (cancer species: $\mathrm{P}=0.617$; ICIs: $\mathrm{P}=0.797$ ). To further explore the sources of heterogeneity, we listed the characteristics of the studies that included OS in Table 2. After comparing the characteristics of each study, we considered that the source of heterogeneity may be related to other lines of treatments that the patient received or patient's TMB cut-off value.

\section{Publication bias}

Ten groups in 12 studies estimated the relationship between TMB level and OS, and we made the egger's test, the result showed that there was no publication bias (Egger's test: $\mathrm{P}=0.247$ ). On the other hand, 16 groups in 13 different studies indicated the relationship between TMB level and PFS, and the funnel plot showed there are no publication bias (Egger's test: $\mathrm{P}=0.411$ ).

\section{Sensitivity analysis}

Sensitivity analysis of high TMB and OS in cancer patients is shown in Figure 6. The ordinate shows 24 groups, whereas the abscissa indicates the pooled HR and 95\% CI. 
Table 1 Characteristics of included trials

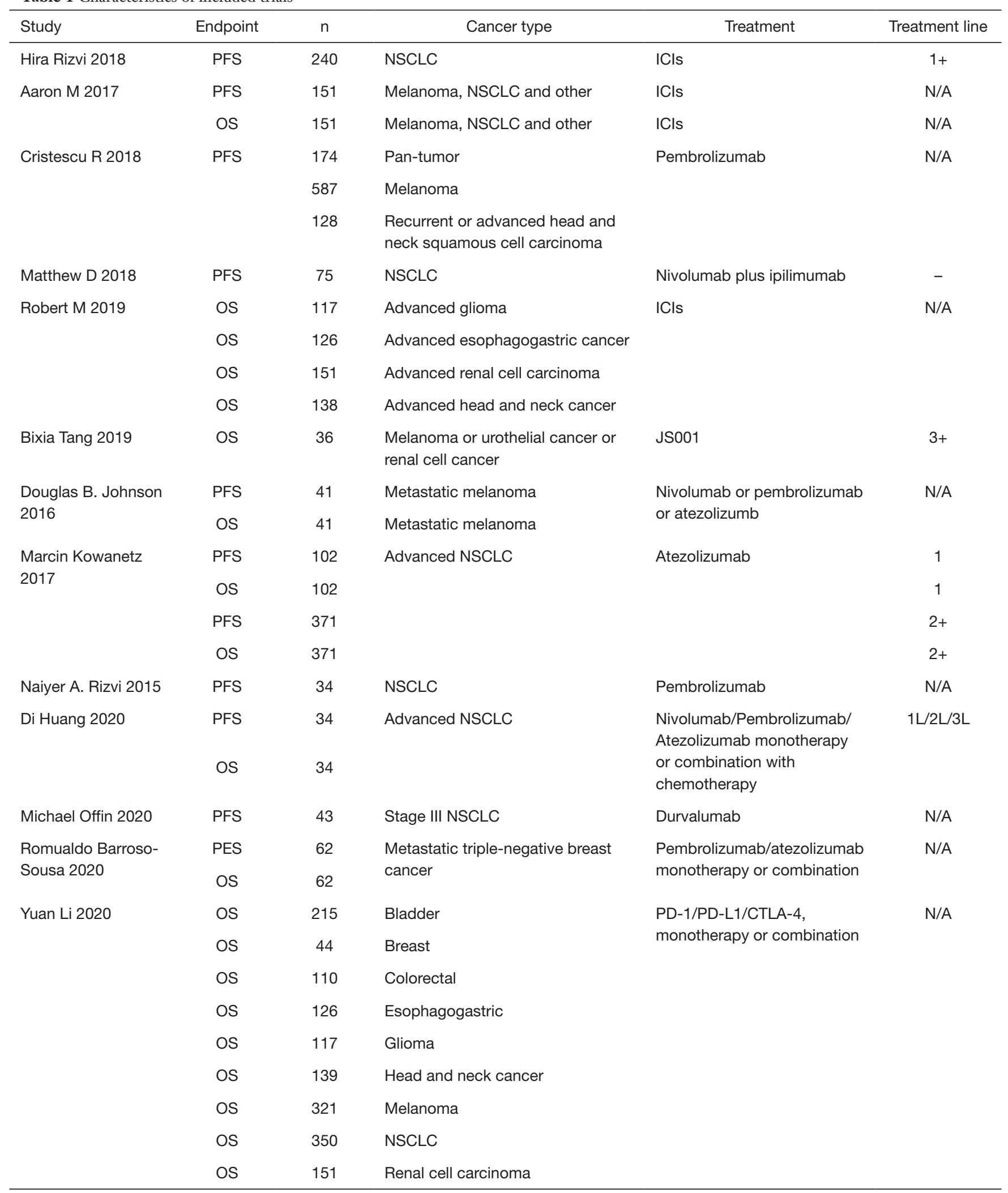

Table 1 (continued) 
Table 1 (continued)

\begin{tabular}{|c|c|c|c|c|c|}
\hline Study & Endpoint & $\mathrm{n}$ & Cancer type & Treatment & Treatment line \\
\hline Jinchul Kim 2020 & PFS & 63 & Advanced gastric & Nivolumab/Pembrolizumab & N/A \\
\hline F. Wang 2019 & OS & 58 & $\begin{array}{l}\text { Advanced gastric cancer (chemo } \\
\text { refractory) }\end{array}$ & Toripalimab & $2+$ \\
\hline \multirow{2}{*}{$\begin{array}{l}\text { Aaron M. Goodman } \\
2020\end{array}$} & PFS & 77 & \multirow{2}{*}{$\begin{array}{l}\text { HSCC, NSCLC, cutaneous } \\
\text { squamous cell carcinoma }\end{array}$} & ICIS & N/A \\
\hline & OS & 77 & & ICIS & N/A \\
\hline
\end{tabular}

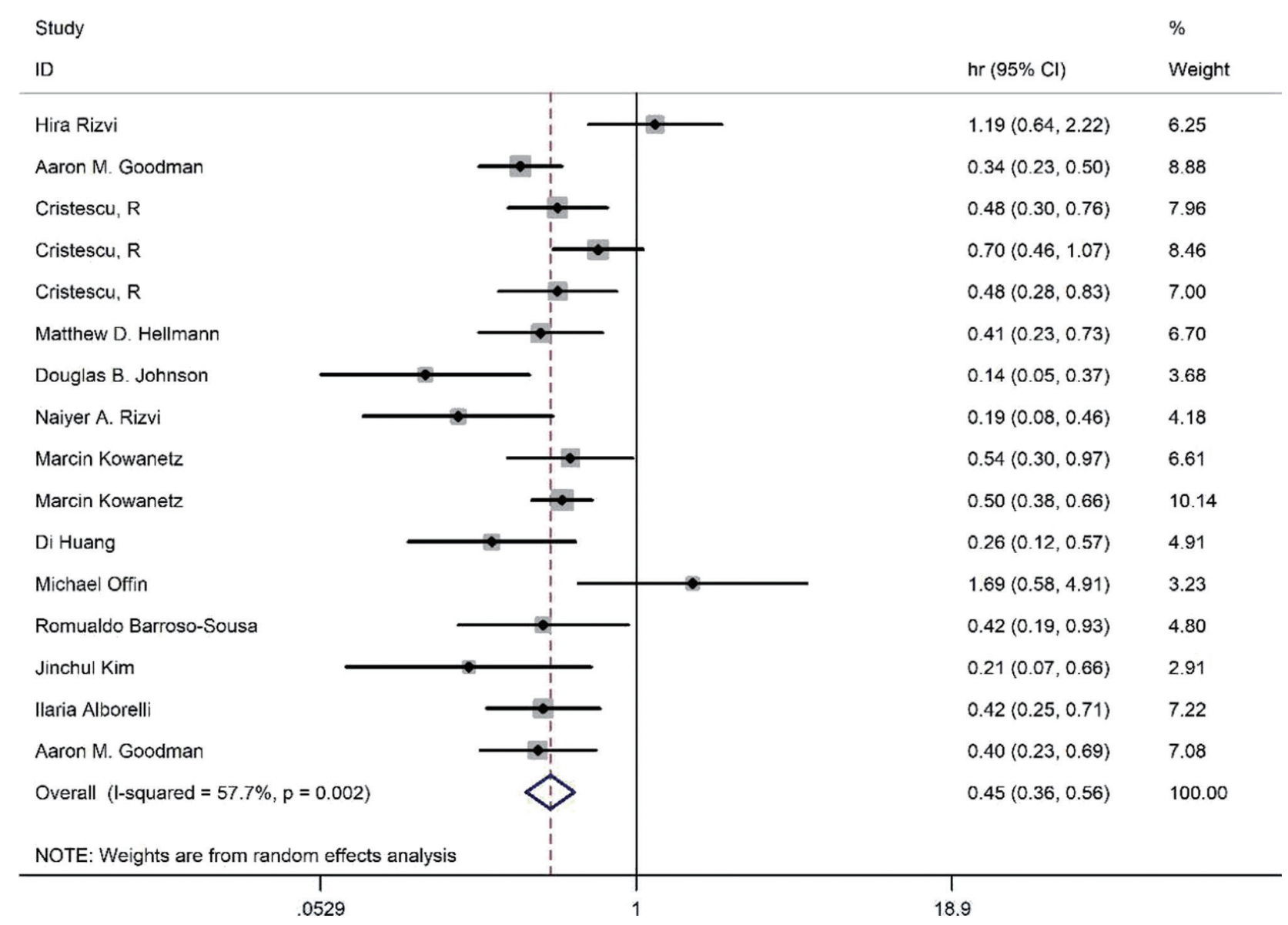

Figure 2 Forest plot for the associations between the progression-free survival of patients and high tumor mutation burden.

The pooled analysis showed that the results were reliable, and each individual study had no significant influence on the total pooled effect. The sensitivity analysis of high TMB and PFS in cancer patients is shown in Figure 7, and the results were reliable.

\section{Discussion}

ICIs include PD-1, PD-L1, and CTAL-4 inhibitors. PD-1 is a transmembrane protein that is mainly expressed in activated T cells, B cells, natural killer (NK) cells, dendritic 


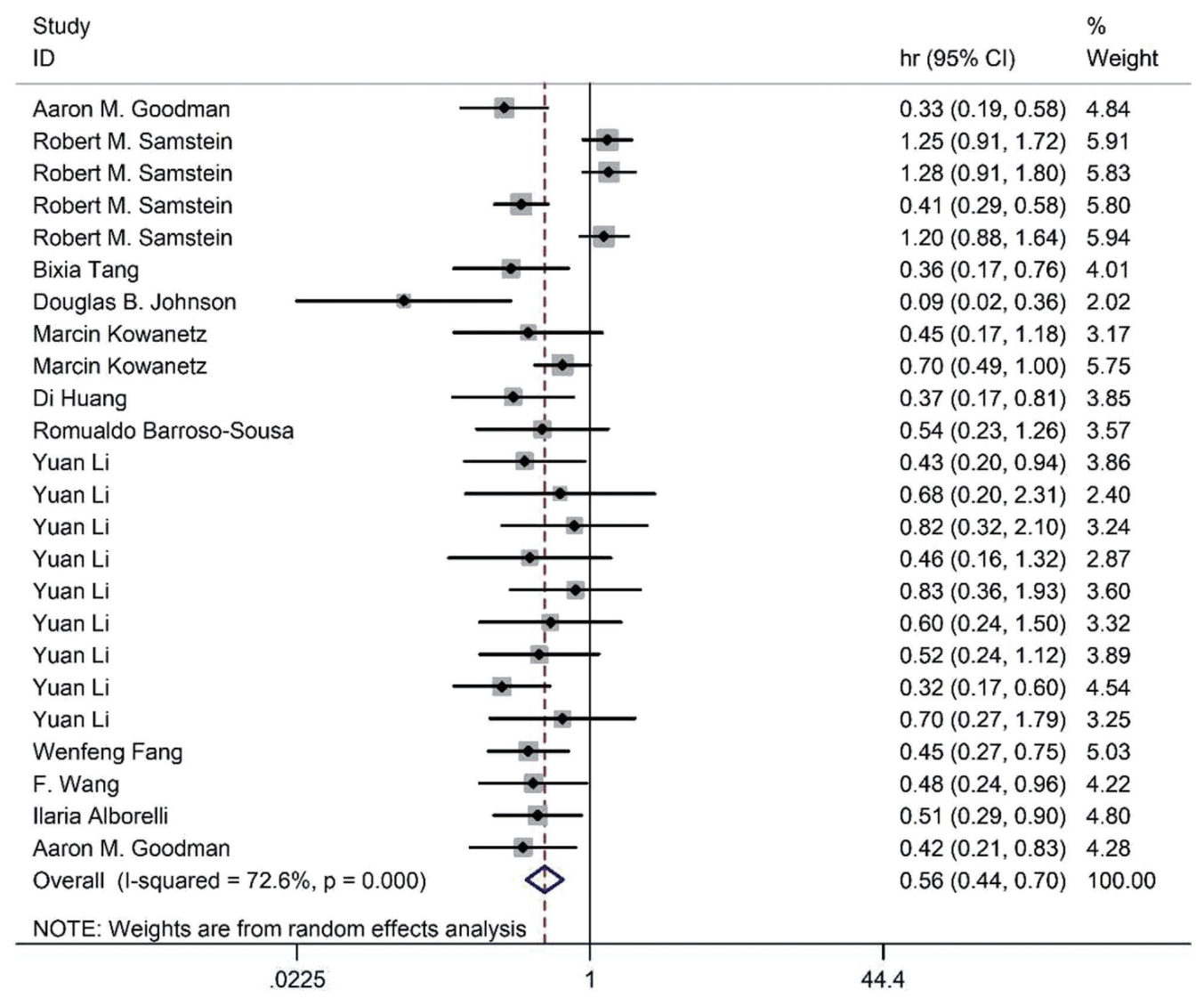

Figure 3 Forest plot for the associations between the overall survival of patients and high tumor mutation burden.

cells, and activated monocytes. Its cognate ligand (PD-L1) is also mainly expressed in the cell membrane of $\mathrm{T}$ cells, B cells, NK cells, macrophages, and other lymphocytes and monocytes. Moreover, PD-L1 is highly expressed in various cancers, such as NSCLC, melanoma, gastric cancer, colon cancer, breast cancer, and pancreatic cancer (28-30). The interaction between PD-L1 and PD-1 can prevent effector T cells from killing cancer cells and promote their immune escape. On the other hand, the protein of CTLA-4 is expressed on the surface of activated $\mathrm{T}$ cells. Cancer cells can activate CTLA-4 and induce the inhibition of the immune response of $\mathrm{T}$ cells, resulting in the immune escape of cancer cells (31). Thus, immunotherapy targeting the PD-1 or CTLA4 pathway could up regulate the antitumor immune effect and result in better prognosis for some cancer patients. In recent years, ICIs have been widely applied in cancer treatment. However, some problems still need to be addressed, such as the low response rate and detection of the sensitive population. To screen out the targeted population, a variety of biomarkers were evaluated, including PD-L1, TMB, deficient mismatch repair, and microsatellite instability (32).

TMB means the total number of non-synonymous mutations. Every 150 non-synonymous mutations can produce 1-2 new antigens. More mutations upregulate the quantity of new antigens. These new antigens are recognized by the autoimmune system as non-autoantigens, resulting in immune response by activating $\mathrm{T}$ cells (5,33-35). Therefore, high TMB could indicate the production of more new antigens than low TMB, suggesting that patients with high TMB are more likely to benefit from ICIs. Many studies have shown that patients with high TMB have a better response rate and prognosis to ICI treatment than those with low TMB $(8,9,36,37)$. Hanna et al. found that for head and neck squamous cell carcinoma, patients with high TMB treated with ICIs had better survival than those with low TMB. Moreover, the data of Carbone DP indicate that TMB and PD-L1 are two independent biomarkers. The TMB level can recognize the beneficiaries better than the expression of PD-L1 in cancer patients treated with 


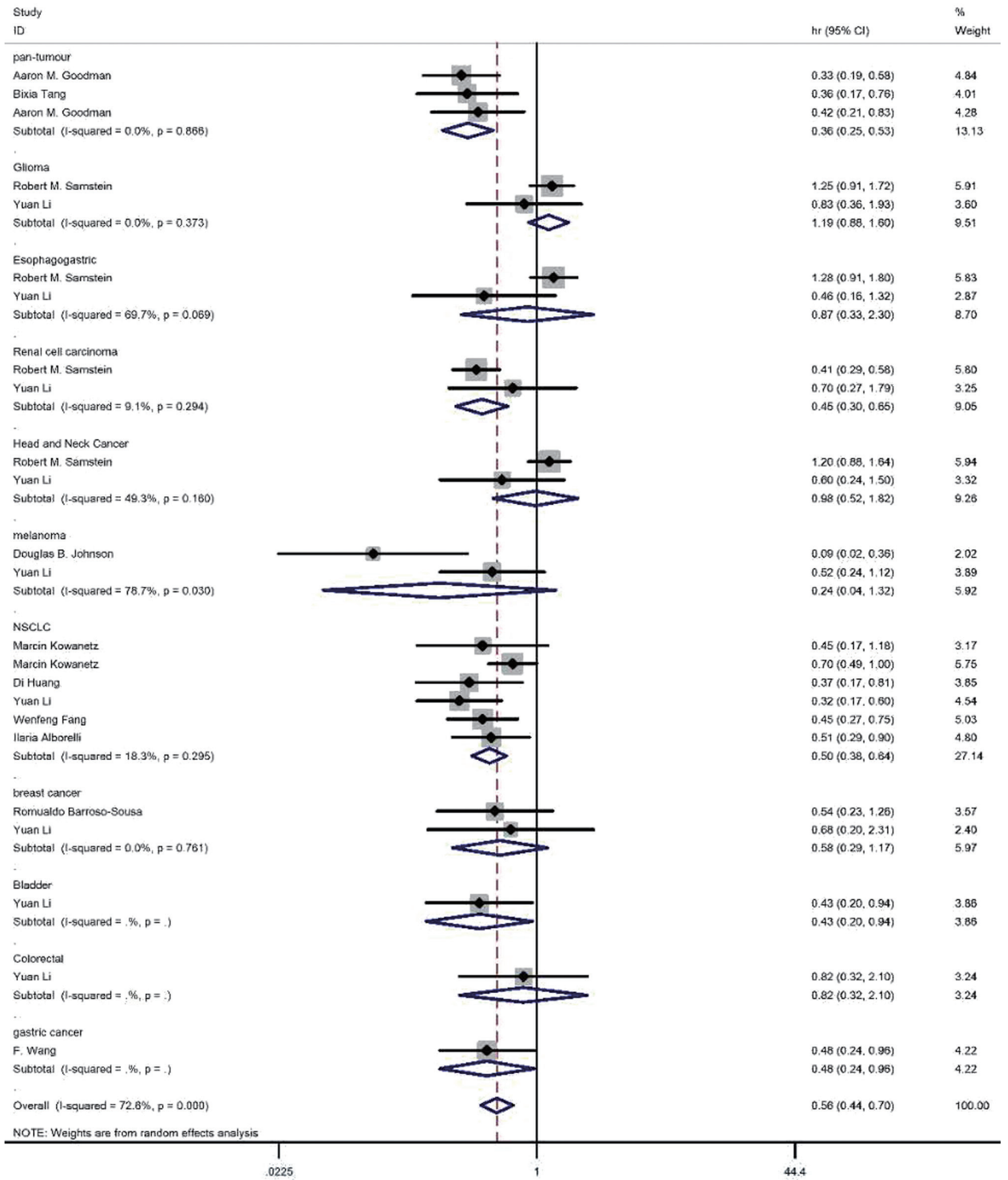

Figure 4 subgroup of meta-analysis about tumor mutation burden and overall survival by cancer type. 


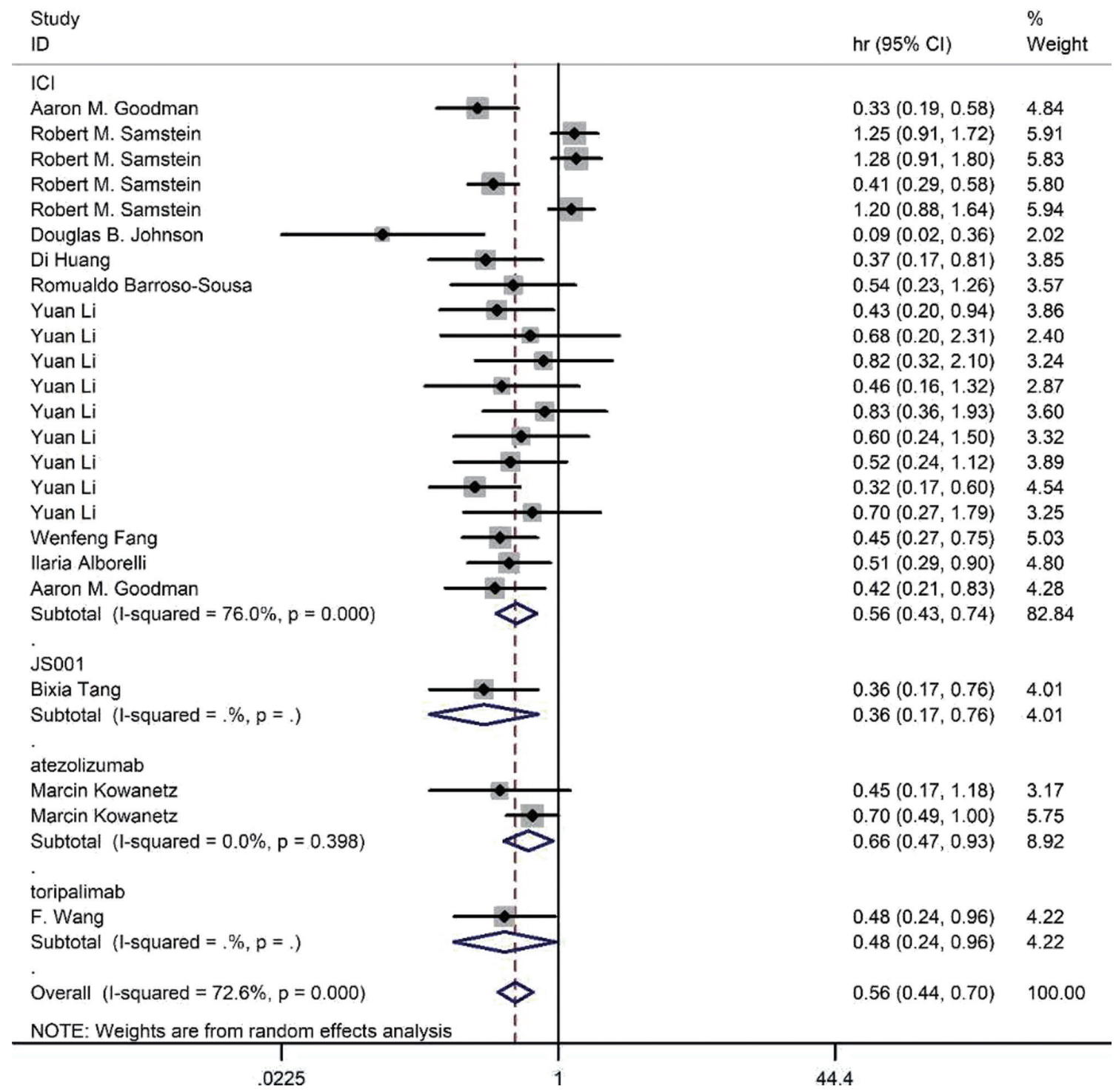

Figure 5 subgroup of meta-analysis about tumor mutation burden and overall survival by ICIs type.

nivolumab (37). In addition, Rizvi et al. found that the TMB level is not proportional to the expression level of PD-L1 (11). Patients with high TMB and PD-L1 expression obtain the best outcomes from ICIs. Thus, high TMB can be used as an independent biomarker in screening cancer patients with low PD-L1 expression who can benefit from ICIs. Moreover, high TMB and PD-L1 expression can be used together to screen patients who can benefit from ICIs.

However, some studies show that high TMB is not related to better response rate and prognosis in cancer patients treated with ICIs (38). Different conclusions have been obtained in terms of the relationship between cancer prognosis and TMB. Thus, whether TMB can be used as a predictive marker of the efficacy of ICIs is still inconclusive. Hence, we conducted this meta-analysis to explore the relationship between TMB and prognosis of cancer patients treated with ICIs and determine whether TMB can be used as a predictive marker for the efficacy of immunotherapy. However, according to the results of our study, we couldn't get the conclusion that high TMB patients had longer OS in pan-tumor because of the high heterogeneity of pooled HR. And the results of regression analysis showed that the types of cancer and ICIs were not sources of high heterogeneity. After analyzing the characteristics of the 
Table 2 The HRs of studies about overall survival

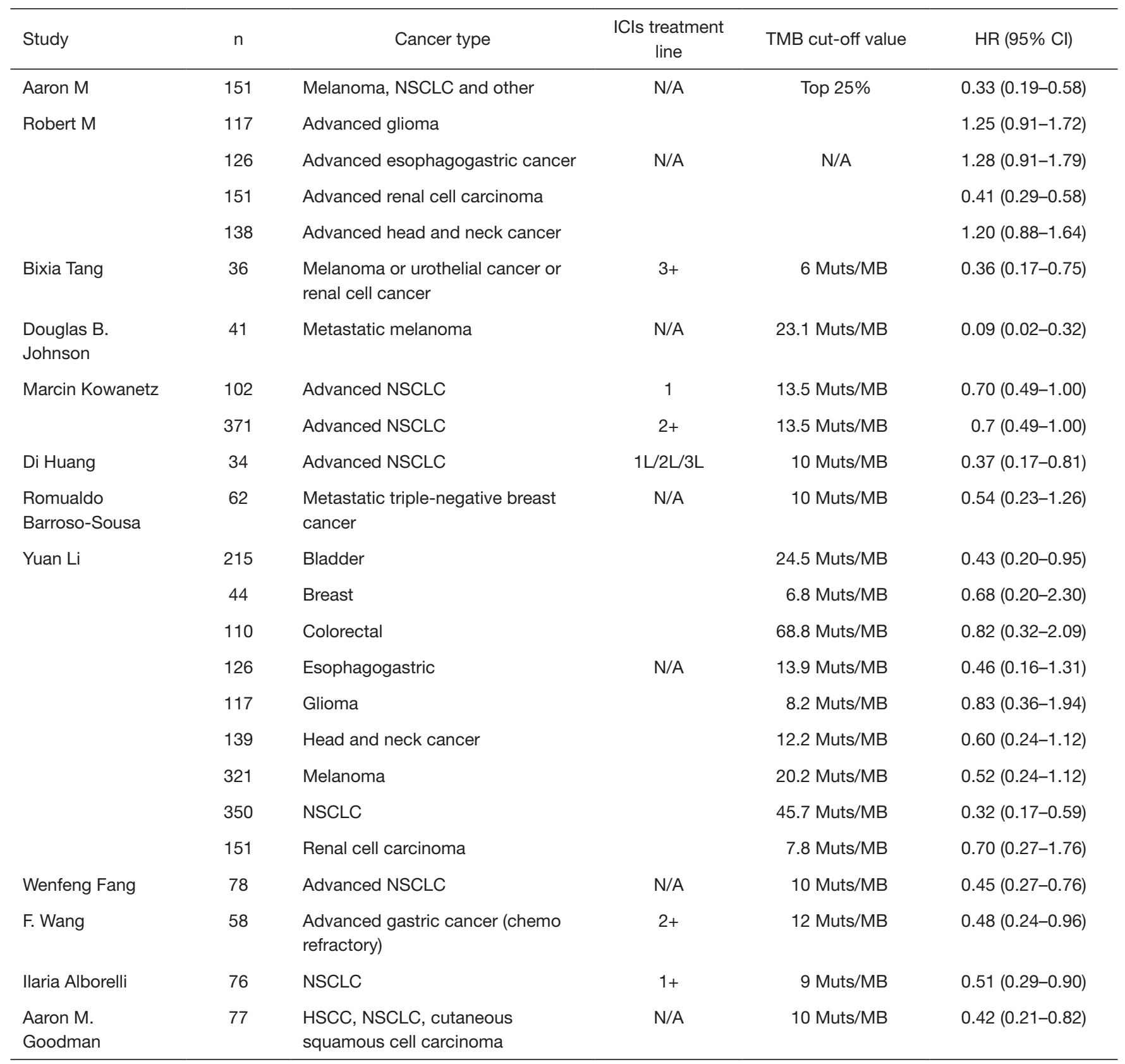

included studies about OS, we considered that the source of heterogeneity may be related to other lines of treatments that the patient received and patient's TMB cut-off value. Meanwhile, we found that high-TMB patients treated with ICIs had better PFS than low-TMB patients. In short, our study shows the TMB can be used as a predictive marker for the efficacy of ICIs because high-TMB patients treated with ICIs had better PFS. However, high TMB patients may not have better OS in pan-tumor.

Although many studies indicate that high TMB is associated with better survival in cancer patients treated with ICIs, this conclusion is not applicable to all cancer types. Samstein et al. explored the relationship between TMB and ICI efficacy in 10 different types of cancers. They found that high-TMB patients with NSCLC, melanoma, and colorectal cancer had better OS with ICI treatment; however, 


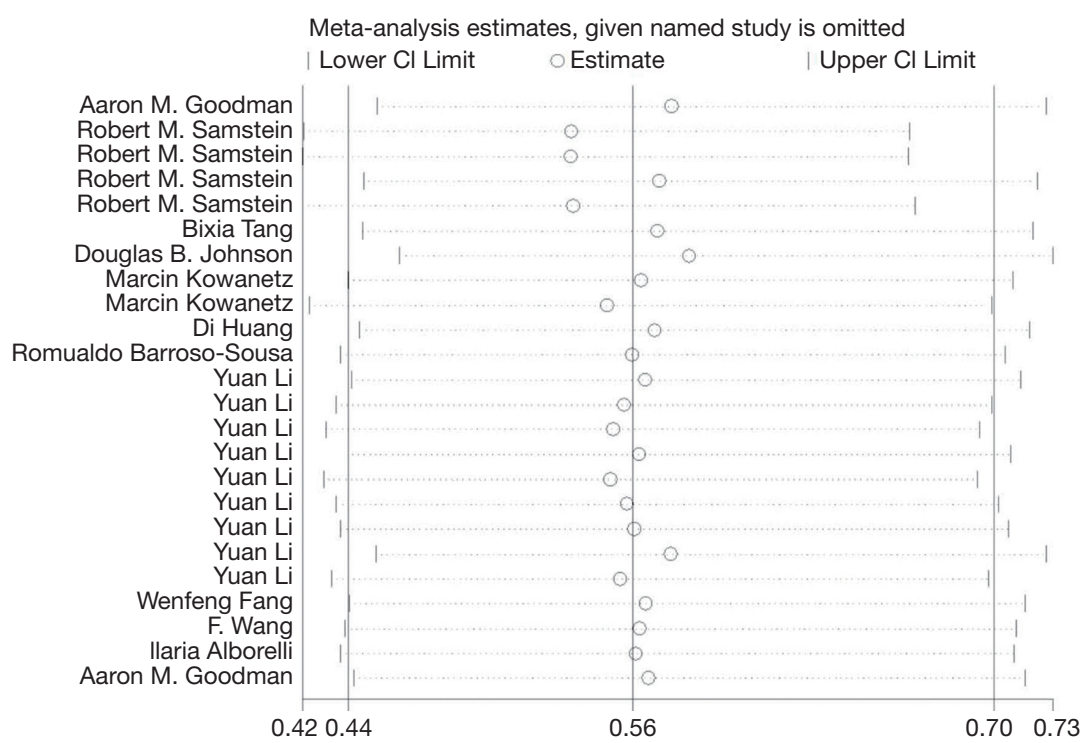

Figure 6 Sensitivity analysis of high tumor mutation burden and overall survival.

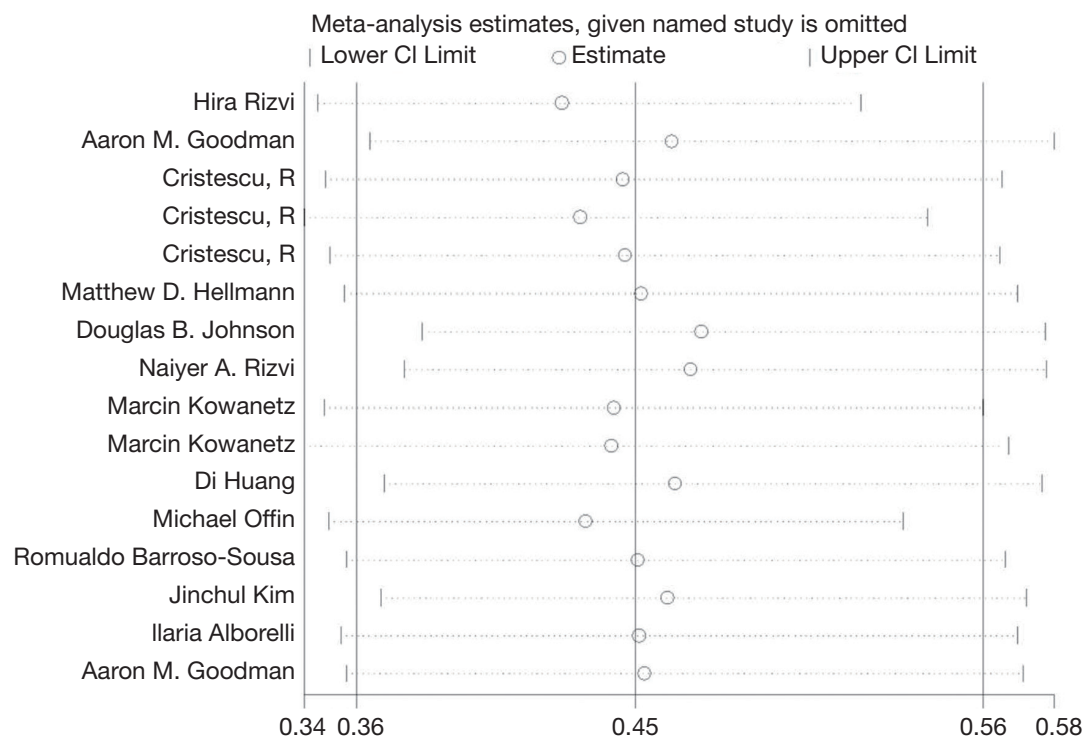

Figure 7 Sensitivity analysis of high tumor mutation burden and progression-free survival.

high-TMB patients with glioma had poor survival (10). Therefore, more clinical studies need to explore the application scope of TMB as an efficacy biomarker of ICIs.

Different studies used different cut-off value of TMB to predictive the efficacy of ICIs, resulting in various conclusions. Hence, the threshold value of TMB as an efficacy biomarker of ICIs should be explored. The MYSTIC (39) clinical trial explored the relationship between the efficacy of ICIs in NSCLC and TMB level in tissue and blood. The study found that for TMB in tissues, patients with $\mathrm{TMB} \geq 10 \mathrm{mut} / \mathrm{Mb}$ had better OS. On the other hand, the critical values for TMB in the blood are divided into $4,8,12,16$, and $20 \mathrm{mut} / \mathrm{Mb}$, and the study found that patients with $\mathrm{TMB} \geq 20 \mathrm{mut} / \mathrm{Mb}$ have better survival. In addition, CheckMate 568 found that for NSCLC patients treated with ICIs, the objective response rate (ORR) no longer increased when $\mathrm{TMB} \geq 10 \mathrm{mut} / \mathrm{Mb}$ (40). Robert et al. explored the critical value of TMB in different 
types of cancers. The critical values of TMB in NSCLC, colorectal cancer, bladder cancer, and melanoma are 13.8, $52.2,17.6$, and $30.7 \mathrm{mut} / \mathrm{Mb}$, respectively. Therefore, the critical value of TMB in each type of cancer still needs to be defined and further explored by a large number of clinical studies.

ICIs are only approved for some types of cancer such as melanoma, bladder cancer, NSCLC, and renal cell carcinoma. In our study, high TMB is beneficial for the PFS of patients, indicating that patients with other types of cancer may benefit from the ICIs. Moreover, one study showed that many types of cancer result in high TMB, which may facilitate the application of ICIs (41). Another study explored the relationship between TMB and ORR. The study included thousands of patients with common cancers. The result showed that the ORR of $55 \%$ of patients is related to TMB, and the correlation coefficient is $0.74(\mathrm{P}<0.001)$. The study predicted that ICIs may be effective for high-TMB patients with sarcomatous lung cancer and basal cell carcinoma (5).

We found a significant heterogeneity between TMB level and $\mathrm{OS}$ of patients in every included study (OS: $\mathrm{P}=0.000$, $\left.\mathrm{I}^{2}=72.6 \%\right)$. Thus, we conducted sensitivity analysis, which showed no significant difference on the result after the removal of each study. The source of heterogeneity may be related to the tumor stage, previous treatment, cut-off value of TMB, etc.

Despite our findings, our study still has some limitations. First, the number of cases included in this study is limited, and more data are needed to confirm our conclusions. Second, because the HR could not be obtained, some studies were excluded, and data were lost. Third, we didn't find the source of high heterogeneity about the metaanalysis of OS. Fourth, the standard of high TMB in some studies is not clear; thus, we did not discuss the specific critical value of TMB as a predictive marker. Finally, each case might have received different treatments before being included in this study, which may lead to different therapeutic effects.

\section{Conclusions}

In conclusion, high TMB is associated with better PFS in patients treated with ICIs. With the further exploration of $\mathrm{TMB}$, it may serve as a predictive marker for the efficacy of ICIs and provide guidance for cancer immunotherapy.

\section{Acknowledgments}

Funding: This study was supported by the Medical Scientific Research Project in Chongqing, China (grant no. 20141003). Chongqing clinical oncological research center, China (grant no. cstc2015yfpt_gcjsyjzx120010). And the Natural Science Foundation Project of Chongqing Science and Technology Commission (CSTC), China (grant no. cstc2018jcyjAX0012).

\section{Footnote}

Reporting Checklist: The authors have completed the PRISMA reporting checklist. Available at http://dx.doi. org/10.21037/tcr-20-1131

Peer Review File: Available at http://dx.doi.org/10.21037/tcr20-1131

Conflicts of Interest: All authors have completed the ICMJE uniform disclosure form (available at http://dx.doi. org/10.21037/tcr-20-1131). The authors have no conflicts of interest to declare.

Ethical Statement: The authors are accountable for all aspects of the work in ensuring that questions related to the accuracy or integrity of any part of the work are appropriately investigated and resolved.

Open Access Statement: This is an Open Access article distributed in accordance with the Creative Commons Attribution-NonCommercial-NoDerivs 4.0 International License (CC BY-NC-ND 4.0), which permits the noncommercial replication and distribution of the article with the strict proviso that no changes or edits are made and the original work is properly cited (including links to both the formal publication through the relevant DOI and the license). See: https://creativecommons.org/licenses/by-nc-nd/4.0/.

\section{References}

1. Ferlay J, Colombet M, Soerjomataram I, et al. Estimating the global cancer incidence and mortality in 2018: GLOBOCAN sources and methods. Int J Cancer 2019;144:1941-53.

2. Bray F, Ferlay J, Soerjomataram I, et al. Global cancer statistics 2018: GLOBOCAN estimates of incidence and 
mortality worldwide for 36 cancers in 185 countries. CA Cancer J Clin 2018;68:394-424.

3. Muenst S, Schaerli AR, Gao F, et al. Expression of programmed death ligand 1 (PD-L1) is associated with poor prognosis in human breast cancer. Breast Cancer Res Treat 2014;146:15-24.

4. Dudnik E, Peled N, Wollner M, et al. MA 02.06 BRAF Mutant NSCLC: Correlation with PD-L1 Expression,TMB, MSI and Response to ICPi and AntiBRAF Therapy. J Thorac Oncol 2017;12:S1804-5.

5. Yarchoan M, Hopkins A, Jaffee EM. Tumor Mutational Burden and Response Rate to PD-1 Inhibition. N Engl J Med 2017;377:2500-1.

6. Krieg C, Nowicka M, Guglietta S, et al. High-dimensional single-cell analysis predicts response to anti-PD-1 immunotherapy. Nat Med 2018;24:144-53.

7. Takada K, Toyokawa G, Shoji F, et al. The Significance of the PD-L1 Expression in Non-Small-Cell Lung Cancer: Trenchant Double Swords as Predictive and Prognostic Markers. Clin Lung Cancer 2018;19:120-9.

8. Hanna GJ, Lizotte P, Cavanaugh M, et al. Frameshift events predict anti-PD-1/L1 response in head and neck cancer. JCI Insight 2018;3:e98811.

9. Goodman AM, Kato S, Cohen PR, et al. Genomic landscape of advanced basal cell carcinoma: Implications for precision treatment with targeted and immune therapies. Oncoimmunology 2017;7:e1404217.

10. Samstein RM, Lee CH, Shoushtari AN, et al. Tumor mutational load predicts survival after immunotherapy across multiple cancer types. Nat Genet 2019;51:202-6.

11. Rizvi H, Sanchez-Vega F, La K, et al. Molecular Determinants of Response to Anti-Programmed Cell Death (PD)-1 and Anti-Programmed Death-Ligand 1 (PD-L1) Blockade in Patients With Non-Small-Cell Lung Cancer Profiled With Targeted Next-Generation Sequencing. J Clin Oncol 2018;36:633-41.

12. Goodman AM, Kato S, Bazhenova L, et al. Tumor Mutational Burden as an Independent Predictor of Response to Immunotherapy in Diverse Cancers. Mol Cancer Ther 2017;16:2598-608.

13. Cristescu R, Mogg R, Ayers M, et al. Pan-tumor genomic biomarkers for PD-1 checkpoint blockade-based immunotherapy. Science 2018;362:eaar3593.

14. Hellmann MD, Nathanson T, Rizvi H, et al. Genomic Features of Response to Combination Immunotherapy in Patients with Advanced Non-Small-Cell Lung Cancer. Cancer Cell 2018;33:843-52.e4.

15. Tang B, Yan X, Sheng X, et al. Safety and clinical activity with an anti-PD-1 antibody JS001 in advanced melanoma or urologic cancer patients. J Hematol Oncol 2019;12:7.

16. Johnson DB, Frampton GM, Rioth MJ, et al. Targeted Next Generation Sequencing Identifies Markers of Response to PD-1 Blockade. Cancer Immunol Res 2016;4:959-67.

17. Kowanetz M, Zou W, Shames D, et al. Tumor Mutation Burden (TMB) is Associated with Improved Efficacy of Atezolizumab in 1L and 2L+ NSCLC Patients. J Thorac Oncol 2017;12:S321-2.

18. Rizvi NA, Hellmann MD, Snyder A, et al. Cancer immunology. Mutational landscape determines sensitivity to PD-1 blockade in non-small cell lung cancer. Science 2015;348:124-8.

19. Huang D, Zhang F, Tao H, et al. Tumor Mutation Burden as a Potential Biomarker for PD-1/PD-L1 Inhibition in Advanced Non-small Cell Lung Cancer. Target Oncol 2020;15:93-100.

20. Offin M, Shaverdian N, Rimner A, et al. Clinical outcomes, local-regional control and the role for metastasis-directed therapies in stage III non-small cell lung cancers treated with chemoradiation and durvalumab. Radiother Oncol 2020;149:205-11.

21. Barroso-Sousa R, Keenan TE, Pernas S, et al. Tumor Mutational Burden and PTEN Alterations as Molecular Correlates of Response to PD-1/L1 Blockade in Metastatic Triple-Negative Breast Cancer. Clin Cancer Res 2020;26:2565-72.

22. Li Y, Chen $Z$, Wu L, et al. Novel tumor mutation score versus tumor mutation burden in predicting survival after immunotherapy in pan-cancer patients from the MSKIMPACT cohort. Ann Transl Med 2020;8:446.

23. Wang F, Wei XL, Wang FH, et al. Safety, efficacy and tumor mutational burden as a biomarker of overall survival benefit in chemo-refractory gastric cancer treated with toripalimab, a PD-1 antibody in phase Ib/II clinical trial NCT02915432. Ann Oncol 2019;30:1479-86.

24. Kim J, Kim B, Kang SY, et al. Tumor Mutational Burden Determined by Panel Sequencing Predicts Survival After Immunotherapy in Patients With Advanced Gastric Cancer. Front Oncol 2020;10:314.

25. Fang W, Ma Y, Yin JC, et al. Comprehensive Genomic Profiling Identifies Novel Genetic Predictors of Response to Anti-PD-(L)1 Therapies in Non-Small Cell Lung Cancer. Clin Cancer Res 2019;25:5015-26.

26. Alborelli I, Leonards K, Rothschild SI, et al. Tumor mutational burden assessed by targeted NGS predicts clinical benefit from immune checkpoint inhibitors in non- 
small cell lung cancer. J Pathol 2020;250:19-29.

27. Goodman AM, Castro A, Pyke RM, et al. MHC-I genotype and tumor mutational burden predict response to immunotherapy. Genome Med 2020;12:45.

28. Böger C, Behrens HM, Mathiak M, et al. PD-L1 is an independent prognostic predictor in gastric cancer of Western patients. Oncotarget 2016;7:24269-83.

29. Que Y, Xiao W, Guan YX, et al. PD-L1 Expression Is Associated with FOXP3 + Regulatory T-Cell Infiltration of Soft Tissue Sarcoma and Poor Patient Prognosis. J Cancer 2017;8:2018-25.

30. Faraj SF, Munari E, Guner G, et al. Assessment of tumoral PD-L1 expression and intratumoral CD8+ T cells in urothelial carcinoma. Urology 2015;85:703.e1-6.

31. Baksh K, Weber J. Immune checkpoint protein inhibition for cancer: preclinical justification for CTLA-4 and PD-1 blockade and new combinations. Semin Oncol 2015;42:363-77.

32. Hause RJ, Pritchard CC, Shendure J, et al. Classification and characterization of microsatellite instability across 18 cancer types. Nat Med 2016;22:1342-50.

33. Gubin MM, Artyomov MN, Mardis ER, et al. Tumor neoantigens: building a framework for personalized cancer immunotherapy. J Clin Invest 2015;125:3413-21.

34. Mansfield AS, Ren H, Sutor S, et al. Contraction of T cell richness in lung cancer brain metastases. Sci Rep 2018;8:2171.

Cite this article as: Wan L, Wang Z, Xue J, Yang H, Zhu Y. Tumor mutation burden predicts response and survival to immune checkpoint inhibitors: a meta-analysis. Transl Cancer Res 2020;9(9):5437-5449. doi: 10.21037/tcr-20-1131
35. Matsushita H, Vesely MD, Koboldt DC, et al. Cancer exome analysis reveals a T-cell-dependent mechanism of cancer immunoediting. Nature 2012;482:400.

36. Necchi A, Anichini A, Raggi D, et al. Pembrolizumab as Neoadjuvant Therapy Before Radical Cystectomy in Patients With Muscle-Invasive Urothelial Bladder Carcinoma (PURE-01): An Open-Label, Single-Arm, Phase II Study. J Clin Oncol 2018;36:3353-60.

37. Carbone DP, Reck M, Paz-Ares L, et al. First-Line Nivolumab in Stage IV or Recurrent Non-Small-Cell Lung Cancer. N Engl J Med 2017;376:2415-26.

38. Dudnik E, Bshara E, Grubstein A, et al. Rare targetable drivers (RTDs) in non-small cell lung cancer (NSCLC): Outcomes with immune check-point inhibitors (ICPi). Lung Cancer 2018;124:117-24.

39. Rizvi N, Barlesi F, Brahmer J, et al. Phase III, randomized, open-label study of durvalumab (MEDI4736) in combination with tremelimumab or durvalumab alone versus platinum-based chemotherapy in first-line treatment of patients with advanced/metastatic NSCLC: MYSTIC. J Immunother Cancer 2015;3:P171.

40. Hellmann MD, Ciuleanu TE, Pluzanski A, et al. Nivolumab plus Ipilimumab in Lung Cancer with a High Tumor Mutational Burden. N Engl J Med 2018;378:2093-104.

41. Chalmers ZR, Connelly CF, Fabrizio D, et al. Analysis of 100,000 human cancer genomes reveals the landscape of tumor mutational burden. Genome Med 2017;9:34. 